\title{
$\bullet$ \\ A Review of the Possible Implication of COVID-19 Lockdown on Eating Habits
}

\section{IJCRR}

Section: Healthcare

Sci. Journal Impact

Factor: 6.1 (2018)

ICV: 90.90 (2018)

(c) (i) (3)

Copyright@IJCRR

\section{Dayanand Peter ${ }^{1}$, P. Xavier ${ }^{2}$}

'Department of Food Processing Technology, Karunya Institute of Technology and Sciences, Coimbatore, India; ${ }^{2}$ Department of Mathematics, Karunya Institute of Technology and Sciences, Coimbatore, India.

\section{ABSTRACT}

The global lockdown due to the recent outbreak of COVID-19 could signal the shift in many a global phenomenon including changes in eating habits around the world. This is because lockdown in itself restricts the availability of foodstuff that are normally available to all at all time. Rather, most purchases during this time are more influenced by consumer income levels and availability of food rather than knowledge on the healthiness of the food itself. However, this period is crucial for many groups of people: for kids because of their increased exposure to television advertisements aimed at the sale of junk foods, those in their adolescence because it's the age of habit development and the wrong habit gained during this time would go on to influence their adult life. In general, it could lead to apparent transmission of epigenetic and/or phenotypic variation down to the next generation cause major changes in lifestyle leading to increased obesity and hyperglycaemia in many countries. This study reviews scientific work in the field of eating habit development, factors influencing these habits, the effect of unhealthy eating habits and its effect on the youth, adults and shift worker. The authors think that physical activity, proper eating habits, nonindulgence in binge eating could positively affect eating habits during the COVID lockdown.

Key Words: Eating habits, COVID-19, Lockdown, Stress eating, Binge eating, Eating frequency

\section{INTRODUCTION}

Eating habits have been a cause of great concern for parents, adults and researchers equally. While parents are constantly worrying about the food choices of their children and the regularity of their diets, adults are more and more conscious of their body and its response to foods ${ }^{1}$. Researchers are in a way trying to find a balance between the thought process of a parent and an adult. They would like to have a perfect world wherein the worlds of Hippocrates food would be a source of medicine to one's body. Over the years, researchers have tried to link food, frequency of eating, eating habits and many other factors to work up the right way of eating ${ }^{2}$. In this review, we would like our readers to get an understanding about eating habits, why it's important to develop the right eating habits, factors that would influence eating habits, effects of unhealthy habits and if the present constraint due to COVID-19 outbreak potentially affect our eating habits.

\section{Eating Habits and the Age of habit development}

It is universally accepted that physical wellbeing is interconnected closely with the food that we eat or on healthy diets ${ }^{3,4}$. However, one among the many major challenge's governmental institutions, nutritionist and promoters of healthy diets face is the question of what interrelated parameters promotes healthier eating. A string of researchers ${ }^{5,6,7,8,9,10}$ over the last decade have been able to link a range of characteristics such as personal preferences, psychological impact, informational, and environmental factors to why people consume a certain food.

The World Health Organization ${ }^{11}$ recognises adolescence as the phase of life when human dietary habits develop. This they say is also the age at which dietary habits are strongly influenced by factors such as socio-cultural, emotional, and behavioural factors. This is also is a phase in human life when they become more independent and have access to foods away from the home environment. However, changes in eating habits, food preferences and shift in the dietary pat-

\section{Corresponding Author:}

Dayanand Peter, Department of Food Processing Technology, Karunya Institute of Technology and Sciences, Coimbatore, India. Email: dayanandpeter@karunya.edu

ISSN: 2231-2196 (Print)

Received: 13.07 .2020
ISSN: 0975-5241 (Online)

Revised: 02.09 .2020
Accepted: 23.09 .2020
Published: 06.10 .2020 
tern are not only an act of individualism and social expressions ${ }^{12,13,14,15}$ but also is an intrinsic factor that can be traced to a person's sense of belonging to a community ${ }^{16}$.

\section{Influencers of Eating Habits}

Research studies on the influence of Malaysian people against the mixed food culture in Malaysia showed that eating reinforces one's social identity because food preferences and preparation is a way of projecting one's sense of community ${ }^{17}$. This idea has perpetuated the system in so much as, traditional practices of food preparation have become and identity and in sense differentiates between them that belong to the community and others, all based on their knowledge or lack thereof of cultural eating practices.

Though eating habits have a cultural twist, much of it is simply based on impulsivity. Impulsivity influences the otherwise multifaceted individual to act without the foresight of potential risks orconsequences ${ }^{18,19}$. Impulsivity is known to manifest in a range of illnesses such as drug use disorder $^{20}$, impulse-control disorder (ICD) $)^{21}$, behavioural addictions $^{22,23,24}$ and Attention-Deficit Hyperactivity Disorder $(\mathrm{ADHD})^{25}$. One of the theories that were used to explain substance use disorder i.e action-to-habit theory suggests that, the cause of most drug use is linked with impulsive intake of drugs that later turned compulsive by excessive indulgence that lead to habit formation ${ }^{26}$.

This action-to-habit theory is a tool to understand eating habits concerning compulsive overeating ${ }^{27}$. In certain western societies where there is a variety of palatable foods readily available, overeating is influenced by the impulsivity of individuals. These splurges of overeating were also linked with prompts such as visual telemarketing that triggered further food craving and splurge-eating. This could ultimately lead to an impulsive action of overeating to transition into a compulsive form of overeating ${ }^{28}$.

Beliefs in food systems differ from culture to culture and also concerning countries. Most Indian and Canadian adolescents believed home-cooked foods to be healthier in terms of micro and macronutrient content ${ }^{29}$. However, rural Indian adolescents also appreciated the importance of contaminant-free food. Opinions varied among this test group when it came down to consuming meat, while Canadian adolescents acknowledged the health impact of meat in their diets, Indian adolescents not so much. The perception of the advantages of healthy eating also varied among respondents, while rural Indians identified improved energy as a benefit of healthy eating, Canadians and urban Indians identified disease prevention as a benefit of healthy eating. Interestingly, some of the barriers identified by both groups of adolescence included peer pressure, obtainability and affordability of unhealthy foods. Academic stress and lack of time were also reported to be barriers for Urban Indians and Canadian girls.
The problems associated with children are a tad different. The modern-day child practices much more power in the decision-making process of a purchase in comparison to children in the past generation ${ }^{30}$. Children today are more forthright and vocal in reasoning with their parents about their requirement for purchasing a product. Recognising this shift in family dynamics, advertisers are creating advertisements targeting children as they know the kind of impact has on product sales. Children consequently spend more time on the television and their sleeping habits are greatlyaffected ${ }^{31}$. Most shifts in eating habit changes can be traced to children watching fast food advertisements on TV amounting to much of the obesity in the children. Some researchers ${ }^{32}$ have proposed a ban on advertisements as a way to check the rising obesity.

Research studies also observed that a students' knowledge of healthy eating habits was rooted in what was in offer in their tables for meals and parents' income levels paid a significant influence on what was on offer ${ }^{33}$. Most parents bought home foods based on their income rather than on their knowledge of healthy foods. Naturally, parents indirectly influenced their children's eating habits as foods placed on the dining table at home turned to be the model healthy eating pattern for most children ${ }^{34}$.

\section{Effects of Unhealthy Eating habits}

In the USA, overweight and obesity rates have dramatically increased leading toa health epidemic of sorts of the current era. Sadly, this has become the case globally and is no more a localised phenomenon ${ }^{35,36}$. Research also recognises obesity as a serious health risk factor which required risk behaviour interventions in young men $^{37}$. In the U.S, for the population aged between 20-39, the incidence rate of obesity has reached $29 \%{ }^{38}$. Studies have identified that among the young, physical inactiveness and unhealthy eating habits contributed to adverse effects on the weight status and their future health as adults ${ }^{39}$.

It can be pointed out that alongside a shift in lifestyles and consumption of junk foods have accounted for most of the health issues including obesity which accounts for more than 300000 deaths annually in the U.S alone. This can be linked to the growing number of fast-food restaurants in the U.S $S^{40}$. On the other hand, studies on the Ukraine Famine of $1932-$ ' $33^{41}$ and the Dutch 'Hunger Winter' Famine of 1944$45^{42}$ suggests an association between prenatal famine exposure and adult type 2 diabetes mellitus. This could mean that there is an apparent possibility of transmission of epigenetic and/or phenotypic variation down the generations if one generation is exposed to prenatal famine exposure. This intergenerational transmission in turn could cause major changes in lifestyle leading to increased obesity and hyperglycaemia ${ }^{43}$. 
How would eating habits affect shift work during a lockdown?

During these altered times of COVID-19, many professionals in the field of medicine such as doctors, nurses and virologists are either working overtime or in altered routines as in the case of most sanitary workers, IT professionals working from home, academicians conducting online classes both in regular working hours as well as the works during odd timings and environments. Apart from the apparent change in shift is the change in routine that doesn't get considered much. This shift could lead to altered routines that disrupt circadian rhythms. The human circadian rhythm adheres to a 24-hour cycle that regulates the normal functioning of our body inclusive of behavioural, endocrine and neurophysiological processes. In a sense, if the circadian rhythm is not in balance it could disrupt our sleep/wake cycle, blood pressure and the release of several hormones. Results from research ${ }^{44}$ suggests that altered sleep patterns could shift the synchrony with light and dark and could disrupt circadian rhythms.

Apart from disruptions circadian rhythms, eating at an odd time, such as late-night eating can alter glucose metabo$\operatorname{lism}^{45}$. Studies have also reported a higher risk to overweight and obesity ${ }^{46}$, metabolic syndrome ${ }^{47}$, type 2 diabetesmellitus $^{48}$ and cardiovascular diseases ${ }^{49}$.

\section{CONCLUSIONS AND RECOMMENDATIONS}

Primary health workers and shift workers whose eating patterns are disrupted during this lockdown could have serious health implications if they do not maintain proper eating habits. Research on shift workers highlighted that the psychological and social health of shift workers can be adversely affected due to the predictable and unconventional shift patterns ${ }^{44}$. In terms of shift regulating in the medical profession, some of the following recommendations from prior research ${ }^{50}$ could be applied to the present times:

- Forward rotation sequence - clockwise direction starting with morning shifts to afternoon shift and ending with nightshifts with no consecutive shifts.

- Restriction of consecutive night shifts to three nights especially for staff keener on the long run at nights to get into a routine.

- The restriction of shift start time to eight or later in the morning to ensure physical and mental freshness of staff.

- Varied shift lengths - night shift to be shorter than day shift as it is virtually impossible to at the same intensity at night. Shift lengths not to exceed 12-hour shifts to ensure the quality and quantity of sleep for staff.

- Regular shift rotas enabling staff to organise their life better.

As theorised through the action-to-habit theory compulsive overeating during the lockdown period could lead to altered unhealthy eating habits. Overeating can be overcome by mixing elements of a food pyramid, Mediterranean pyramid or MyPlate system. The most recent Mediterranean associate's for example includes social aspects of happiness that come from cultural and environmental often associated with homemade and traditional foods. They also point out the use of locally and seasonally available agricultural produces and products $^{51}$.

Lack of physical activity and unhealthy dietary habits during this lockdown period could lead to potentially adverse effects on weight status in our young leading them to an unhealthy adulthood future. The World Heath Organisation${ }^{52}$ recommendations for physical activity can be adhered to by all age groups.

Those in the age group of 5-17 years:

- Minimum 60 minutes of moderate to vigorous-intensity physical activity every day.

- Vigorous-intensity activities should be aimed at strengthening muscle and bone and done at least thrice a week.

Those in the age group 18-64 years:

1. $150 \mathrm{~min}$. of moderate-intensity or $75 \mathrm{~min}$. of vigorousintensity physical activity throughout the week.

2. Muscle-strengthening activities for every major muscle groups twice a week.

3. Aerobic activity in 10 minutes bouts.

Those in the age group 65 years and above:

1. 150 minutes of moderate-intensity physical activity spread across the week.

2. The physical activity aimed at enhancing balance to prevent falls for people with low mobility on 3 days in a week.

3. Muscle-strengthening activities for every major muscle groups twice a week.

4. Aerobic activity in 10 minutes bouts.

Availability, access, affordability and undue pressure through advertisements of unhealthy foods could lead to binge eating. Studies point out that there is a need to work on personal core low self-esteem and emotional regulation among binge-eater to reduce binge eating ${ }^{53}$. Therefore, this period of lockdown can be used to improve the self-esteem and build the emotional balance of our kids.

It would be wise to remember that there is always a possibility of transmission of epigenetic and/or phenotypic variation through generations ${ }^{43}$. It should also be realised that the lockdown period in many countries is similar to the situation faced during famine exposure. The similarities would include the lack of food security and availability and accessibility to essentials food items and as such could lead to increased obesity and hyperglycaemia globally. 


\section{REFERENCE}

1. Niba Johnson, Noufeena D. Y., Parvathi, Priya Joseph, Priya Reshma Aranha, Asha P. Shetty. A study on knowledge regarding the prevention of iron deficiency anaemia among adolescent girls in selected pre-university colleges of mangaluru. International Journal of Current Research and Review, 2016; 8 (18): 5-7.

2. Ong, C., Phuah, K. Y., Salazar, E., \& How, C. H. Managing the 'picky eater' dilemma. Singapore medical journal, 2014;55(4), 184-190.

3. N.A. Georgiou, J. Garssen, R.F. Witkamp. Pharma-nutrition interface: the gap is narrowing ear. J. Pharmacol., 2011; 651,1-8.

4. Willett, W. C.Diet and health: what should we eat? Science. 1994;(22); 264(5158): 532-537.

5. Willett, W. C., \&Stampfer, M. J. Current evidence on healthy eating. Annual Review of Public Health, 2013; 34, 77-95.

6. De Ridder, D., De Vet, E., Stok, M., Adriaanse, M., \& De Wit, J. Obesity, overconsumption and self-regulation failure: The unsung role of eating appropriateness standards. Health Psychology Review, 2012; 7, 146-165.

7. Ikeda, N., Saito, E., Kondo, N., Inoue, M., Ikeda, S., Satoh, T., Shibuya, K. What has made the population of Japan healthy? The Lancet, 2011; 378, 1094-1105.

8. Michie, S., Abraham, C., Whittington, C., McAteer, J., \& Gupta, S. Effective techniques in healthy eating and physical activity interventions: A meta-regression. Health Psychology, 2009; 28, 690-701.

9. Traill, W. B.Policies to promote healthy eating in Europe: A structured review of policies and their effectiveness. Nutrition Reviews, 2012; 70, 188-200.

10. Wansink, B., Payne, C. R., \& Shimizu, M. "Is this a meal or snack?" Situational cues that drive perceptions. Appetite, 2010; 54, 214-216.

11. World Health Organization. Nutrition in adolescence: issues and challenges for the health sector: issues in adolescent health and development. Geneva: WHO, 2005.

12. Mennell, S.All Manners of Food. Chicago: University of Illinois Press, 1996.

13. Mintz, S.W.Tasting Food, Tasting Freedom: Excursions into Eating, Culture and the Past. Boston: Beacon Press, 1996.

14. Gabaccia, D.We Are What We Eat: Ethnic Food and the Making of Americans. Cambridge, MA: Harvard University Press, 1998.

15. Anderson, E.N.Everyone Eats Understanding Food and Culture. New York: New York University Press, 2005.

16. Fischler, C.Commensality, Society and Culture. Social Science Information, 2011;50 (3-4), 528-548.

17. Raymond L. M. Lee. Malaysian Identities and Mélange Food Cultures, Journal of Intercultural Studies, 2017;38 (2), 139-154.

18. Dalley, J., Everitt, B., \& Robbins, T. Impulsivity, Compulsivity, and Top-Down Cognitive Control. Neuron, 2011;69(4), 680694.

19. Fineberg, N., Chamberlain, S., Goudriaan, A., Stein, D., Vanderschuren, L., Gillan, C., Shekar, S., Gorwood, P., Voon, V., Morein-Zamir, S., Denys, D., Sahakian, B., Moeller, G., Robbins, T., \& Potenza, M. New developments in human neurocognition: clinical, genetic, and brain imaging correlates of impulsivity and compulsivity, CNS Spectrums, 2014; 19(1), 68-89.

20. Grant, J., \& Chamberlain S. Impulsive action and impulsive choice across substance and behavioural addictions: Cause or consequence? Addictive Behaviors, 2014; 39(11), 1632-1639.

21. Grant, J., \& Potenza, M. Compulsive aspects of impulse control disorders, Psychiatr Clin North Am, 2006; 29(2), 539-551.

22. Blanco, C., Potenza, M., Won Kim, S., Ibáñez, A., Zaninelli, R., Saiz-Ruiz, J., \& Grant, J. A pilot study of impulsivity and com- pulsivity in pathological gambling. Psychiatry Research, 2009; 167(1-2), 161-168.

23. Grant, J., Potenza, M., Weinstein, A., \& Gorelick, D.Introduction to behavioural addictions, Am J Drug Alcohol Abuse, 2010; 36(5), 233-241.

24. Robbins, T., \& Clark, L.Behavioral addictions. Current Opinion in Neurobiology, 2015; 30(0), 66-72.

25. Linhartová, P., Látalová, A., Barteček, R., Širůček, J., Theiner, P., Ejova, A., Kašpárek, T. Impulsivity in patients with borderline personality disorder: A comprehensive profile compared with healthy people and patients with ADHD. Psychological Medicine, 2019; 1-10.

26. Everitt, B., \& Robbins, T. Drug addiction: Updating actions to habits to compulsions ten years on. Annual Review of Psychology, 2015; Aug 7doi: 10.3389/fpsyg.2015.01608.

27. Robbins, T., Gillan, C., Smith, D., de Wit, S., \& Ersche, K. D. Neurocognitive endophenotypes of impulsivity and compulsivity: towards dimensional psychiatry. Trends in Cognitive Sciences, 2012; 16(1), 81-91.

28. Smith, D., \& Robbins, T.The neurobiological underpinnings of obesity and binge eating: a rationale for adopting the food addiction model. Biol Psychiatry, 2013; 73(9), 804-810.

29. Correa N., Rajaraman D., Swaminathan S., Vaz M., Jayachitra K.G., Lear S.A. \&Punthakee Z., Perceptions of healthy eating amongst Indian adolescents in India and Canada, Appetite, 2017;DOI: 10.1016/j.appet.2017.05.029.

30. Amit Gosar, Dina Rajput and Jigna Ganatra. Advertisements Targeting Children, Asian Journal of Education and Social Studies. 2018; 2(4): 1-15, 2018; Article no. AJESS.44536.

31. Van den Bulck J.Television viewing, computer game playing, and internet use and self-reported time to bed and time out of bed in secondary-school children. Sleep. 2004; 27(1):101-104.

32. Dhar T, Baylis K. Fast food consumption and the ban on advertising targeting children. The quebee experience. Journal of Marketing Research. 2011; 48(5):799-813.

33. KaziEnamul Hoque, KaziFardinul Hoque and Revethy A/P Thanabalan. Relationships between parents' academic backgrounds and incomes and building students' healthy eating habits. PeerJ2018; DOI 10.7717/peerj.4563.

34. Vollmer RL, Baietto J. Practices and preferences: exploring the relationships between food-related parenting practices and child food preferences for high fat and/or sugar foods, fruits, and vegetables. Appetite 2017; 113:134-140.

35. Mancino, L.; Kinsey, J. Dietary Knowledge Enough? Hunger, Stress, and Other Roadblocks to Healthy Eating; USDA, Economic Research Service: Washington, DC, USA, 2008.

36. LaCaille, L.J.; Nichols Dauner, K.N.; Krambeer, R.J.; Pedersen, J. Psychosocial and Environmental Determinants of Eating Behaviors, Physical Activity, and Weight Change Among College Students: A Qualitative Analysis. J. Am. Coll. Health. 2011; 59, 531-538.

37. Ashton, L.M.; Hutchesson, M.J.; Rollo, M.E.; Morgan, P.J.; Collins, C.E. Motivators and Barriers to Engaging in Healthy Eating and Physical Activity: A Cross-Sectional Survey in Young Adult Men. Am. J. Men's Health 2017; 11, 330-343.

38. Ogden, C.L.; Carroll, M.; Kit, B.; Flegal, K. Prevalence of obesity among adults: the United States, 2011-2012.NCHS Data Brief 2013; 131, 1-8.

39. Allom, V. and Mullan, B.Maintaining healthy eating behaviour: Experiences and perceptions of young adults. Nutr. Food Sci. 2014; 44, 156-167.

40. Ashakiranand Deepthi R Kolar, "Fast food and their impacts on health", Journal of khrishna institute of medical sciences university review of the literature: JKIMSU, 2012 Vol.1 No.2 JulyDec. 
41. Lumey, L. H., Khalangot, M. D. and Vaiserman, A. M. Association between type 2 diabetes and prenatal exposure to the Ukraine famine of 1932-1933: a retrospective cohort study. Lancet Diabetes Endocrinol. 2015; 3, 787-794.

42. ACJ Ravelli, JHP van der Meulen, RPJ Michels, C Osmond, DJP Barker, CN Hales, OP Bleker, Glucose tolerance in adults after prenatal exposure to famine, The Lancet,1998; Volume 351, Issue 9097,173-177.

43. Chihua Li, Elmar W. Tobi, Bastiaan T. Heijmans and L. H. Lumey. The effect of the Chinese Famine on type 2 diabetes mellitus epidemics. Nat Rev Endocrinol 2019; 15, 313-314.

44. Fiona M. Nea, L. Kirsty Pourshahidi, John M. Kearney, M. Barbara E. Livingstone, Carolina Bassul, Clare A. Corish A qualitative exploration of the shift work experience: the perceived effect on eating habits, lifestyle behaviours and psychosocial wellbeing Journal of Public Health, 2018; 1-11, doi:10.1093/ pubmed/fdy047.

45. Grant CL, Coates AM, Dorrian J, Kennaway DJ, Wittert GA, Heilbronn LK, Pajcin M, Della Vedova C, Gupta CC, Banks $\mathrm{S}$. Timing of food intake during simulated night shift impact glucose metabolism: a controlled study. Chronobiol Int., 2017; 34(8):1003-1013.

46. Antunes, L., Levandovski, R., Dantas, G., Caumo, W., \& Hidalgo, M. Obesity and shift work: Chronobiological aspects. Nutrition Research Reviews, 2010; 23(1), 155-168.
47. Wang F, Zhang L, Zhang Y, Zhang B, He Y, Xie S, Li M, Miao X, Chan EY, Tang JL, Wong MC, Li Z, Yu IT, Tse LA.A metaanalysis on night shift work and risk of metabolic syndrome. Obes Rev. 2014;15: 709-720. 10.1111/obr.12194.

48. Pan A, Schernhammer ES, Sun Qi, Frank B. Hu. Rotating night shift work and risk of type 2 diabetes: two prospective cohort studies in women. PLoS Med 2011;8(12):e1001141.

49. Haupt CM, Alte D, Dorr M, Robinson DM, Felix SB, John U, Völzke H. The relation of exposure to shift work with atherosclerosis and myocardial infarction in a general population. Atherosclerosis 2008;201(1):205-11.

50. Wilson J.L. The impact of shift patterns on healthcare professionals. Journal of Nursing Management, 2002; 10, 211-219.

51. Willett, W.C., Sacks, F., Trichopoulou A., Drescher G., FerroLuzzi A., Helsing E., Trichopoulos D.Mediterranean diet pyramid: a cultural model for healthy eating, The American Journal of Clinical Nutrition, Volume 61, Issue 6, June 1995, 1402S1406S, https://doi.org/10.1093/ajen/61.6.1402S.

52. World Health Organization. Diet, nutrition and the prevention of chronic diseases WHO Technical Report Series, No. 916 (TRS 916), 2003.

53. Amy L. Burton and Maree J. Abbott. Processes and pathways to binge eatings: development of an integrated cognitive and behavioural model of binge eating, Journal of Eating Disorders. 2019; 7:18https://doi.org/10.1186/s40337-019-0248-0. 